\title{
Prevalence and Antimicrobial Susceptibility Pattern of Extended-Spectrum Beta-Lactamase-Producing Enterobacteriaceae in the United Arab Emirates
}

\author{
Mansour Al-Zarouni ${ }^{a} \quad$ Abiola Senok $^{b}$ Fatima Rashid $^{a}$ \\ Shaikha Mohammed Al-Jesmi ${ }^{a}$ Debadatta Panigrahi ${ }^{\text {b }}$ \\ ${ }^{a}$ Al Qassimi Hospital Laboratory Sharjah, Ministry of Health, and ${ }^{b}$ Department of Clinical Sciences, \\ College of Medicine, University of Sharjah, Sharjah, United Arab Emirates
}

\section{Key Words}

Antimicrobial resistance $\cdot$ Enterobacteriaceae .

Escherichia coli $\cdot$ Klebsiella pneumoniae $\cdot$ Extended

spectrum $\beta$-lactamase

\begin{abstract}
Objective: To investigate the prevalence and antibiotic susceptibility pattern of extended-spectrum $\beta$-lactamases (ESBL)-producing Enterobacteriaecae among patients in the United Arab Emirates. Materials and Methods: A total of 130 Enterobacteriaceae comprising of Escherichia coli $(n=83)$, Klebsiella pneumoniae $(n=45)$ and Klebsiella oxytoca $(n=2)$ was studied. Of these 130 isolates, 64 were from urine. ESBL screening was by disc diffusion and confirmatory tests for ESBL phenotype were conducted using BD Phoenix ${ }^{T M}$ ESBL System and cephalosporin/clavulanate combination discs. Susceptibility to a panel of antibiotics was evaluated. $\boldsymbol{R e}$ sults: Of the 130 isolates, 53 (41\%) were identified as having ESBL phenotype; of these, 32 (60\%) were E. coli, 20 (36\%) K. pneumoniae and 2 (4\%) $K$. oxytoca. ESBL phenotype was seen in $100 \%$ of endotracheal tubes isolates, 20 (31\%) from urine, 7 (58\%) from blood and 4 (80\%) from catheter tips. Amikacin susceptibility was $100 \%$. Over $90 \%$ of ESBL isolates showed resistance to aztreonam and cephalosporins. All Klebsiella isolates were carbapenem sensitive. One ESBL isolate showed intermediate resistance to imipenem and meropenem (both
\end{abstract}

MIC $8 \mu \mathrm{g} / \mathrm{ml}$ ), cefotetan (MIC $32 \mu \mathrm{g} / \mathrm{ml}$ ) and piperacillin/ tazobactam (MIC $32 \mu \mathrm{g} / \mathrm{ml}$ ). MIC for the carbapenems was lower in non-ESBL isolates $(0.034 \mu \mathrm{g} / \mathrm{ml})$ than $\mathrm{ESBL}$ isolates $(0.071 \mu \mathrm{g} / \mathrm{ml})$. Resistance to gentamicin, ciprofloxacin and piperacillin/tazobactam was higher in ESBL than non-ESBL isolates $(p<0.05)$. Conclusion: A high prevalence of ESBLproducing bacteria exists among in-patients in the United Arab Emirates. Amikacin and carbapenems remain the most effective drugs, but the presence of carbapenem-resistant ESBL-producing E. coli and occurrence of multidrug resistance are of concern. Continued surveillance and judicious antibiotic usage are recommended.

Copyright $\odot 2007$ S. Karger AG, Basel

\section{Introduction}

The upward trend in the prevalence of pathogens producing extended-spectrum $\beta$-lactamases (ESBL) is of increasing clinical concern. Infections with these ESBLproducing organisms continue to be associated with higher rates of mortality, morbidity and health care costs. ESBL arise as a result of mutations in the TEM-1, TEM-2, or SHV-1 genes which are commonly found in the Enterobacteriaceae family [1]. The mutation causes an alteration of their amino acid configuration making these enzymes capable of hydrolyzing a wider spectrum of

\section{KARGER}

Fax +4161306 1234

E-Mail karger@karger.ch

www.karger.com
(C) 2007 S. Karger AG, Basel

1011-7571/08/0171-0032\$24.50/0

Accessible online at:

www.karger.com/mpp
Dr. Abiola Senok

Department of Clinical Sciences

College of Medicine, University of Sharjah

PO Box 27272, Sharjah (United Arab Emirates)

Tel. +971 6505 7220, Fax +9716558 5879, E-Mail asenok@sharjah.ac.ae 
$\beta$-lactam antibiotics including penicillins, oxyiminocephalosporins, such as cefotaxime (CTX), ceftazidime (CAZ), and ceftriaxone, as well as monobactams, e.g. azetreonam. However, these plasmid-mediated enzymes have no detectable activity against carbapenems and their activity is inhibited by clavulanic acid (CA) $[1,2]$. Although ESBLs occur predominantly in Klebsiella species and Escherichia coli, they have also been described in other genera of the Enterobacteriaceae, family including Citrobacter, Serratia, Proteus, Salmonella, and Enterobacter $[1,2]$. Additionally, other types of ESBL such as CTX-M and OXA type enzymes have now been described $[1,3,4]$. ESBL-producing strains often exhibit a multidrug-resistant phenotype, including resistance to aminoglycosides and fluoroquinolones, further limiting the therapeutic options available to the clinician $[5,6]$.

The first description of ESBL-producing organisms was reported in 1983 [7] and since then reports from Europe, the USA and the Far East have confirmed the role of ESBL-producing organisms in nosocomial infections [1]. However, the prevalence of ESBL among clinical isolates varies among geographic areas with low rates of 3$8 \%$ in Sweden, Japan and Singapore to much higher prevalence rates reported from Portugal: $34 \%$, Latin America: $30-60 \%$, and Turkey: $58 \%[1,8,9]$. This study presents the first reported data describing the prevalence and antibiotic susceptibility pattern of ESBL-producing Enterobacteriaecae among patients in the United Arab Emirates (UAE).

\section{Materials and Methods}

\section{Bacterial Strains}

The study was carried out at the Microbiology Laboratory, AlQassimi Hospital, Sharjah, United Arab Emirates from April 2005 to May 2006. Specimens/isolates were obtained from in-patients in six general hospitals in the UAE. Duplicate isolates from the same patient was not allowed, hence only one isolate was accepted per episode of infection. A total of 130 Enterobacteriaceae comprising E. coli: 83, Klebsiella pneumoniae: 45, and Klebsiella oxytoca: 2 isolated during the collection period were included in the study. Of the 130 isolates, 64 were from urine and the proportion obtained from different specimen types are listed in table 1. Identification of isolates was done based on cultural characteristics and reactions in standard biochemical tests.

Detection of ESBL

In keeping with the Clinical and Laboratory Standards Institute (CLSI) recommended guidelines [10], screening for ESBL was performed by disc diffusion using $\mathrm{CAZ}$ and cefpodoxime; confirmatory tests for ESBL phenotype were carried out using BD Phoenix $^{\mathrm{TM}}$ ESBL Automated System (Becton, Dickinson, Md., USA) and cephalosporin/clavulanate combination discs. These
Table 1. Number of Enterobacteriaceae isolated from different specimen types

\begin{tabular}{lc}
\hline Specimen & Isolates \\
\hline Urine & 64 \\
Blood & 12 \\
Pus & 12 \\
High vaginal swab & 10 \\
Sputum & 9 \\
Wound & 7 \\
Endotracheal tubes/secretion & 6 \\
Catheter tip & 5 \\
Ear/eye secretions & 3 \\
Cerebral spinal fluid & 1 \\
Umbilical cord & 1 \\
\hline Total & 130 \\
\hline
\end{tabular}

tests were applied to all 130 isolates. The Phoenix ${ }^{\mathrm{TM}}$ ESBL test used fixed concentrations of the following drugs or drug combinations: cefpodoxime, CAZ, CAZ plus CA, CTX plus CA, and ceftriaxone plus CA. The isolates were subcultured on MacConkey agar to obtain a pure culture from which a $0.5 \mathrm{McF}$ arland suspension was obtained and tested according to the manufacturer-provided protocol. Phenotypic confirmation with antibiotic disks containing a combination of cephalosporin plus CA in conjunction with a corresponding cephalosporin disc alone was carried out and interpreted according to CLSI guidelines [10]. Discs used were CAZ $(30 \mu \mathrm{g})$, CAZ plus CA (CAZ/CA, 30/10 $\mu \mathrm{g})$, CTX (30 $\mu \mathrm{g})$ and CTX plus CA (CTX/CA, 30/10 $\mu \mathrm{g})$ all obtained from Becton Dickinson, USA. Briefly, isolates were subcultured on MacConkey agar and 0.5 McFarland standard prepared from the pure colonies was inoculated on Muller Hinton agar plates. Cephalosporin discs (CAZ and CTX) and cephalosporin with CA discs (CAZ/CA and CTX/CA) were applied on the inoculated plates and incubated in ambient air at $35^{\circ} \mathrm{C}$ for 16 to $18 \mathrm{~h}$. Following incubation, the diameter of the zones of inhibition was measured and $\mathrm{a} \geq 5-\mathrm{mm}$ increase in the zone of inhibition for the CAZ/CLA and CTX/CLA-containing disc versus the corresponding CAZ or CTX disc was considered positive for ESBL.

\section{Antibiotics Susceptibility}

The susceptibility of the isolates to a panel of antibiotics was evaluated using Vitek I (bioMérieux, Vitek Inc, Hazelwood, Mo., USA), BD Phoenix ${ }^{\mathrm{TM}}$ and Etest (AB Biodisk, Solna, Sweden). The antibiotic susceptibility of the isolates to the following agents was tested: amikacin, aztreonam, ciprofloxacin, gentamicin, cefotetan, cefuroxime, ceftriaxone, CAZ, CTX, imipenem, meropenem and piperacillin/tazobactam. E. coli ATCC 25922 was used as a negative control. The tests for the automated Vitek I and BD Phoenix system were carried out following manufacturer-recommended protocol. The Vitek cards for identification (GNI) and susceptibility testing (GNS-203, GNS108) were used. For the Etest, isolates were subcultured on MacConkey agar to obtain a pure culture. A 0.5 McFarland suspension was prepared from the pure culture and used to inoculate Muller Hinton agar plate prior 
Table 2. Distribution of ESBL and non-ESBL producers among E. coli and Klebsiella spp. isolates

\begin{tabular}{lcc}
\hline Isolate & ESBL & Non-ESBL \\
\hline E. coli $(\mathrm{n}=83)$ & $32(39 \%)$ & $51(61 \%)$ \\
K. pneumoniae $(\mathrm{n}=45)$ & $19(42 \%)$ & $26(58 \%)$ \\
K. oxytoca $(\mathrm{n}=2)$ & $2(100 \%)$ & $0(0 \%)$ \\
\hline
\end{tabular}

to application of Etest strips. Following incubation in inverted position at $35^{\circ} \mathrm{C}$ for $16-20 \mathrm{~h}$, the MIC level was read and interpreted in accordance with CLSI guidelines.

\section{Results}

With the exception of one E. coli isolate, there was $100 \%$ agreement between the two confirmatory tests for ESBL detection. This isolate was determined to be ESBL positive by BD Phoenix and CTX +/- CLAV but nonESBL by CAZ +/- CLAV. Of the 130 isolates, 53 (41\%) were identified as having ESBL phenotype, of which 32 (60\%) were E. coli, 19 (36\%) K. pneumoniae and 2 (4\%) K. oxytoca. The two K. oxytoca isolates were ESBL producers. The proportion of ESBL phenotypes for each microorganism is given in table 2 . All six isolates obtained from endotracheal tubes showed ESBL phenotype. Additionally, 20/64 (31\%) of isolates obtained from urine specimens, 7/12 (58\%) from blood and 4/5 (80\%) from catheter tips were found to be ESBL producers. In contrast, no ESBL-positive strain was obtained from high vaginal swab.

There was $100 \%$ susceptibility to amikacin. Of the 53 ESBL-producing isolates, 48 (90\%) showed resistance to aztreonam (MIC $\geq 64 \mu \mathrm{g} / \mathrm{ml}$ ) and cephalosporins with only two ESBL isolates being sensitive to CAZ (MIC $\geq 1 \mu \mathrm{g} / \mathrm{ml}$ ) by all testing methods. With the exception of one non-ESBL $K$. pneumoniae and one ESBL E. coli isolates both with MIC $>32 \mu \mathrm{g} / \mathrm{ml}$, all other isolates were sensitive to cefotetan. All K. pneumoniae and K. oxytoca isolates were sensitive to imipenem and meropenem. However, two E. coli isolates (one ESBL and one nonESBL) were resistant to these drugs. The ESBL isolate which showed intermediate resistance to imipenem and meropenem (MIC by Etest: $8 \mu \mathrm{g} / \mathrm{ml}$ for both drugs) also showed intermediate resistance to cefotetan (MIC $32 \mu \mathrm{g} /$ $\mathrm{ml}$ ) and piperacillin/tazobactam (MIC $32 \mu \mathrm{g} / \mathrm{ml}$ ). Overall, the MIC for the carbapenems was lower in non-ESBL isolates compared with ESBL isolates (0.034 vs. $0.071 \mu \mathrm{g} /$ $\mathrm{ml}$, respectively). A significantly higher proportion of ESBL-producing isolates compared to non-ESBL producers were found to be resistant to gentamicin (67 vs. 6\%), ciprofloxacin (38 vs. 19\%) and piperacillin/tazobactam (25 vs. $2.8 \%) ; \mathrm{p}<0.05$.

\section{Discussion}

The presence of ESBL enables certain Gram-negative bacteria to inactivate extended-spectrum (third-generation) cephalosporins, broad-spectrum penicillins and monobactams but do not affect the cephamycins or carbapenems. As the presence of these enzymes significantly impacts the efficacy of $\beta$-lactam therapy, there is a need for clinical laboratories to accurately recognize ESBL producers so as to better support therapy and provide accurate prevalence data. The findings presented here indicate that there is a high prevalence of ESBL-producing Enterobacteriaceae circulating among hospitalized patients in the UAE. Global reports have shown that considerable differences exist in the occurrence and proportion of ESBL-producing isolates in different countries [1, 8, 9, 11-16]. In Europe, a large-scale survey of intensive care units found that the prevalence of ESBL in Klebsiella ranged from a low of 3\% in Sweden to as high as 34\% in Portugal [8]. Higher figures of 30-60\% have been reported for the South American countries of Brazil, Venezuela and Colombia [11-13]. In Asia, National Surveys have indicated the presence of ESBLs in 5-8\% of E. coli isolates from Korea, Japan, Malaysia and Singapore with higher rates of up to $24 \%$ for other Asian countries $[1,9]$. ESBL production in Klebsiella have also been reported to be as low as $5 \%$ in Japan and Australia with higher rates of $20-50 \%$ in other parts of the continent $[1,9,14]$. In the Arabian Gulf region, 7.5\% of Enterobacteriaceae and Pseudomonas isolated over a 1-year period in Kuwait were reported as being ESBL producers using the VITEK 2 system [15]. Thus, comparative to other surveys, the finding of $41 \%$ ESBL producers in this study is on the higher end of the spectrum. A predominance of either $K$. pneumoniae or E. coli has often been reported among the ESBL isolates identified in different geographical regions. A prevalence of ESBL-producing K. pneumoniae versus ESBL-producing E. coli (70 vs. $28.6 \%$ ) has been reported in Pakistan [16], and in Italy a 2003 nationwide survey found that the most prevalent ESBL-positive species among hospitalized patients was E. coli, a switch from the predominance of K. pneumoniae in 1999 [17]. In contrast to these other reports, equal proportions of K. pneumo- 
niae and E. coli were identified among the ESBL-producing isolates in our setting. Similar to other reports [18], the majority of Enterobacteriaceae isolates were from urine specimens, indicating the need for active screening of urine cultures for ESBL producers. Indeed, the detection of EBSL producers in urine has been described as representing an epidemiologic marker of colonization and potential for transfer between patients [1]. However, as high proportions of isolates obtained from other sources were found to be ESBL producers, it is imperative that active screening of Enterobacteriaceae isolates from all specimen types should be adopted to address the high prevalence rate of ESBL isolates. Unfortunately, many clinical microbiological laboratories still face significant problems with ESBL screening and identification as ESBL pathogens can present with variations in the in vitro pattern of resistance to $\beta$-lactam agents. Proficiency-testing studies performed by the World Health Organization and the Centers for Disease Control have raised concerns about the current ability of many clinical laboratories to detect ESBL-producing microorganisms [19, 20]. Some enzymes (TEM-3 and SHV-2) confer high levels of resistance to cephalosporins, whereas others, such as TEM-7 and TEM-12, confer low levels of resistance, which possibly makes it even more difficult to detect them through the susceptibility tests routinely used in microbiology laboratories. In this study, the CLSI protocol was applied with satisfactory results thus indicating that this approach of screening and phenotypic confirmation integrating both automated and manual methods is applicable in our setting and useful for incorporation into routine microbiological testing.

The antimicrobial susceptibility results show that amikacin, imipenem and meropenem were the most effective antibiotics against the ESBL producers. Although carbopenems are widely regarded as the drugs of choice for treatment of infection caused by ESBL-producing organisms, production of $\beta$-lactamases capable of hydrolyzing carbapenems has been reported in Enterobacteriaceae mostly in Enterobacter spp. and Serratia spp. [21, 22]. Carbapenem resistance also arises from the production of large quantities of chromosomal and plasmid-mediated cephalosporinases combined with decreased drug permeability through the outer membrane [23]. In E. coli, although the first report of carbapenem resistance appeared in 1999 [24], the occurrence remains a rare phenomenon and only a few cases of carbapenem-resistant $E$. coli strains have been documented in the literature [23]. We identified an ESBL-producing E. coli isolate which was resistant to the carbapenems, piperacillin-

ESBL-Producing Enterobacteriaceae in the United Arab Emirates tazobactam and cefotetan. It has been suggested that such low-level resistance to imipenem may arise from AmpC hyperproduction and loss of porins [23-25]. There is also the potential role of variants of the original ESBL enzymes such as CTX-M $\beta$-lactamases and even efflux changes giving rise to carbapenem resistance [20]. New findings indicate that the spread of CTX-M type ESBLs, especially in E. coli, may provide a favorable background for selection of carbapenem resistance [26]. While this study was aimed at determining the prevalence of ESBL isolates in our setting, we also recognize the need for the molecular characterization of these isolates, particularly in light of our findings. Although we have not carried out the molecular characterization of this carbapenem-resistant $E$. coli, the finding of such resistant isolate in our setting is a serious cause for concern, indicative of the need for more aggressive surveillance and evaluation of the molecular epidemiology and characterization of ESBL isolates in the UAE. Hence, we are embarking on further work to determine the molecular characterization of ESBL-producing isolates circulating in the UAE.

ESBL-producing organisms often show cross-resistance with non- $\beta$-lactam antibiotics, such as aminoglycosides and quinolones, resulting in limitation of therapeutic options. A significantly higher level of resistance to ciprofloxacin, gentamicin and piperacillin-tazobactam was demonstrable in the ESBL-producing isolates, indicating their limited use in the treatment of infections due to these pathogens. This co-resistance arises probably because these plasmid-mediated enzymes are transferable between bacterial species and are also capable of incorporating genetic material coding for resistance to other antibiotics. Indeed plasmid-mediated quinolone resistance has been reported in K. pneumoniae and $E$ coli, associated with acquisition of the qnr gene [27]. Tazobactam is an inhibitory compound which is expected to hinder the activity of ESBL. However, the usefulness of $\beta$ lactam $/ \beta$-lactamase inhibitor combinations for the treatment of infections caused by ESBL-producing organisms remains vague and failures have been reported. Our findings echo these concerns as $25 \%$ of ESBL isolates exhibited resistance to piperacillin-tazobactam.

\section{Conclusion}

This study shows a high prevalence of ESBL in hospitalized patients in the UAE occurring in equal proportion among E. coli and K. pneumoniae isolates. Amikacin and carbapenems remain the most useful drugs for treat- 
ment of ESBL infections. However, the findings of a carbapenem-resistant ESBL-producing E. coli isolate as well as the occurrence of multidrug resistance are of major concern. Large surveys, continued surveillance by clini- cal microbiology laboratories, judicious use of antimicrobial agents as well as implementation of infection control measures are recommended if the frequency of ESBL isolates is to be reduced in this setting.

\section{References}

1 Paterson DL, Bonomo RA: Extended-spectrum beta-lactamases: a clinical update. Clin Microbiol Rev 2005;18:657-686.

$\checkmark 2$ Bradford PA: Extended-spectrum beta-lactamases in the 21st century: characterization, epidemiology, and detection of this important resistance threat. Clin Microbiol Rev 2001;14:933-951.

-3 Bonnet R: Growing group of extended-spectrum beta-lactamases: the CTX-M enzymes. Microb Agents Chemother 2004;48:1-14.

-4 Mugnaioli C, Luzzaro F, De LF, Brigante G, Perilli M, Amicosante G, Stefani S, Toniolo A, Rossolini GM: CTX-M-type extendedspectrum beta-lactamases in Italy: molecular epidemiology of an emerging countrywide problem. Antimicrob Agents Chemother 2006;50:2700-2706.

5 Diaz PQ, Bello HT, Dominguez MY, Trabal NF, Mella SM, Zemelman RZ, Gonzalez GR: Resistance to gentamicin, amikacin and ciprofloxacin among nosocomial isolates of Klebsiella pneumoniae subspecies producing extended spectrum beta-lactamases (in Spanish). Rev Med Chil 2004;132:11731178.

6 Paterson DL, Mulazimoglu L, Casellas JM, Ko WC, Goossens H, Von GA, Mohapatra S, Trenholme GM, Klugman KP, McCormack JG, Yu VL: Epidemiology of ciprofloxacin resistance and its relationship to extendedspectrum beta-lactamase production in Klebsiella pneumoniae isolates causing bacteremia. Clin Infect Dis 2000;30:473-478.

7 Knothe H, Shah P, Krcmery V, Antal M, Mitsuhashi S: Transferable resistance to cefotaxime, cefoxitin, cefamandole and cefuroxime in clinical isolates of Klebsiella pneumoniae and Serratia marcescens. Infection 1983;11: 315-317.

-8 Hanberger H, Garcia-Rodriguez JA, Gobernado $\mathrm{M}$, Goossens $\mathrm{H}$, Nilsson LE, Struelens MJ: Antibiotic susceptibility among aerobic gram-negative bacilli in intensive care units in 5 European countries. French and Portuguese ICU Study Groups. JAMA 1999;281: 67-71.

9 Lewis MT, Yamaguchi K, Biedenbach DJ, Jones RN: In vitro evaluation of cefepime and other broad-spectrum beta-lactams in 22 medical centers in Japan: a phase II trial comparing two annual organism samples. The Japan Antimicrobial Resistance Study Group. Diagn Microbiol Infect Dis 1999;35: 307-315.
10 National Committee for Clinical Laboratory Standards: Performance standards for antimicrobial susceptibility testing; 15th informational supplement (M100-S15). Wayne, National Committee for Clinical Laboratory Standards, 2005.

11 Otman J, Cavassin ED, Perugini ME, Vidotto MC: An outbreak of extended-spectrum beta-lactamase-producing Klebsiella species in a neonatal intensive care unit in Brazil. Infect Control Hosp Epidemiol 2002;23:8-9.

12 Pfaller MA, Jones RN, Doern GV: Multicenter evaluation of the antimicrobial activity for six broad-spectrum beta-lactams in Venezuela: comparison of data from 1997 and 1998 using the Etest method. Venezuelan Antimicrobial Resistance Study Group. Diagn Microbiol Infect Dis 1999;35:153158.

13 Sader HS, Gales AC, Granacher TD, Pfaller MA, Jones RN: Prevalence of antimicrobial resistance among respiratory tract isolates in Latin America: results from SENTRY antimicrobial surveillance program (1997-98). Braz J Infect Dis 2000;4:245-254.

-14 Bell JM, Turnidge JD, Gales AC, Pfaller MA, Jones RN: Prevalence of extended spectrum beta-lactamase (ESBL)-producing clinical isolates in the Asia-Pacific region and South Africa: regional results from SENTRY Antimicrobial Surveillance Program (1998-99). Diagn Microbiol Infect Dis 2002;42:193198.

15 Jamal W, Rotimi VO, Khodakhast F, Saleem R, Pazhoor A, Al Hashim G: Prevalence of extended-spectrum beta-lactamases in Enterobacteriaceae, Pseudomonas and tenotrophomonas as determined by the VITEK 2 and $\mathrm{E}$ test systems in a Kuwait teaching hospital. Med Princ Pract 2005;14:325-331.

16 Shah AA, Hasan F, Ahmed S, Hameed A: Prevalence of extended spectrum B-lactamases in nosocomial and outpatients (ambulatory). Pak J Med Sci 2004;19:187-191.
17 Luzzaro F, Mezzatesta M, Mugnaioli C, Perilli M, Stefani S, Amicosante G, Rossolini GM, Toniolo A: Trends in production of extended-spectrum beta-lactamases among Enterobacteria of medical interest: report of the second Italian nationwide survey. J Clin Microbiol 2006;44:1659-1664.

18 Dimitrov TS, Udo EE, Emara M, Awni F, Passadilla R: Etiology and antibiotic susceptibility patterns of community-acquired urinary tract infections in a Kuwait hospital. Med Princ Pract 2004;13:334-330.

19 Hageman JC, Fridkin SK, Mohammed JM, Steward CD, Gaynes RP, Tenover FC: Antimicrobial proficiency testing of National Nosocomial Infections Surveillance System hospital laboratories. Infect Control Hosp Epidemiol 2003;24:356-361.

-20 Tenover FC, Mohammed MJ, Stelling J, O'Brien T, Williams R: Ability of laboratories to detect emerging antimicrobial resistance: proficiency testing and quality control results from the World Health Organization's external quality assurance system for antimicrobial susceptibility testing. J Clin Microbiol 2001;39:241-250.

21 Nordmann P, Poirel L: Emerging carbapenemases in Gram-negative aerobes. Clin Microbiol Infect 2002;8:321-331.

22 Poirel L, Nordmann P: Acquired carbapenem-hydrolyzing beta-lactamases and their genetic support. Curr Pharm Biotechnol 2002;3:117-127.

-23 Poirel L, Heritier C, Spicq C, Nordmann P: In vivo acquisition of high-level resistance to imipenem in Escherichia coli. J Clin Microbiol 2004;42:3831-3833.

24 Stapleton PD, Shannon KP, French GL: Carbapenem resistance in Escherichia coli associated with plasmid-determined CMY-4 beta-lactamase production and loss of an outer membrane protein. Antimicrob Agents Chemother 1999;43:1206-1210.

-25 Odeh R, Kelkar S, Hujer AM, Bonomo RA, Schreckenberger PC, Quinn JP: Broad resistance due to plasmid-mediated AmpC betalactamases in clinical isolates of Escherichia coli. Clin Infect Dis 2002;35:140-145.

-26 Lartigue M-F, Poirel L, Poyart C, RéglierPoupet H, Nordmann P: Ertapenem resistance of Escherichia coli. Emerg Infect Dis 2007;13:315-317.

27 Paterson DL: Resistance in gram-negative bacteria: Enterobacteriaceae. Am J Infect Control 2006;34:S20-S28. 